\title{
Quality of experience aware network selection model for service provisioning in heterogeneous network
}

\author{
Nagaraja Gadde ${ }^{1}$, Basavaraj Jakkali ${ }^{2}$, Ramesh Babu Halasinanagenahalli Siddamallaih ${ }^{3}$, \\ Gowrishankar ${ }^{2}$ \\ ${ }^{1}$ Department of Information Science and Engineering, SJC Institute of Technology, Chickballapur, India \\ ${ }^{2}$ Department of Computer Science and Engineering, BMS College of Engineering, Bangalore, India \\ ${ }^{3}$ Department of Computer Science and Engineering, Sai Vidya Institute of Technology Bangalore, India
}

\begin{tabular}{l} 
Article Info \\
\hline Article history: \\
Received Mar 31, 2021 \\
Revised Aug 13, 2021 \\
Accepted Sep 10, 2021 \\
\hline
\end{tabular}

Keywords:

Decision making

Handover

Heterogeneous network

MADM

Quality of experience

RAT selection

\begin{abstract}
Heterogeneous wireless networks (HWNs) are capable of integrating the different radio access technologies that make it possible to connect mobile users based on the performance parameters. Further quality of service (QoS) is one of the major topics for HWNs, moreover existing radio access technology (RAT) methodology are designed to provide network QoS criteria. However, limited work has been carried out for the RAT selection mechanism considering user QoS preference and existing models are developed based on the multi-mode terminal under a given minimal density network. For overcoming research issues this paper present quality of experience (QoE) RAT (QOE-RAT) selection methodology, incorporating both network performance criteria and user preference considering multiple call and multi-mode HWNs environment. First, this paper presents fuzzy preference aware weight (FPAW) and multi-mode terminal preference aware TOPSIS (MMTPA-TOPSIS) for choosing the best RAT for gaining multiservices. Experiment outcomes show the QOE-RAT selection method achieves much superior packet transmission outcomes when compared with state-of-art Rat selection methodologies.
\end{abstract}

This is an open access article under the CC BY-SA license.

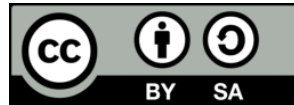

\section{Corresponding Author:}

Nagaraja Gadde

Department of Information Science and Engineering, SJC Institute of Technology

Chickballapur-562101, Karnataka, India

Email: nagarajgadde11@gmail.com

\section{INTRODUCTION}

In recent days, robots or autonomous driving vehicle has gained fine attention due to capability of information dissemination, efficiency, and traffic safety, most of the autonomous vehicle like Google car [1] is mainly based on the perception model which includes the several machine intelligence and onboard sensors for maneuvering on-street along with other vehicles. Moreover, autonomous vehicles (AV) are improvised through computing infrastructure and networking based on the intelligence transportation system (ITS). Since AV possesses many limitations of efficiency, perception sensors, and driving safety in a holistic approach mainly relies on low latency and reliability [2]. Furthermore, to support the requirement, several radio access technologies (RATs) have been developed along with the various transmission characteristics such as frequency, delay, bandwidth, and signal coverage. For instance, the universal mobile telecommunication (UMT)-model provides a huge range of lower bandwidth and signal coverage. further models such as comprehensive $r$ archive network (CRAN) [3], worldwide interoperability for microwave access (WiMax), and long term evolution (LTE) uses transmission technologies like orthogonal frequency-division multiplexing (OFDM) and multiple in multiple out (MIMO) for improvising the data 
transmission rate and spectral efficiency and provides a huge signal coverage range [4]. Furthermore, wireless local area network (WLAN) or local area network that is based on the IEEE standard of 801.1 provides data transmission at high speed along with restricted coverage [5].

Moreover, considering the cellular network coverage, various networks are deployed which form heterogeneous wireless networks (HWNs) along with overlapping signal coverage area [6], further mutual integration and co-existence of the various wireless networks through the various access technologies has shown promising result. Since wireless network transmission possesses differences in diversity in user services and performance, it is more important to be dependent on the selection of algorithm for providing an efficient connection service for users, for instance multimode terminals (MT) in a heterogeneous wireless network environment [7]. Further, vertical handoff (VHO) has been one of the hottest decision algorithms in a heterogeneous wireless network. Moreover, while designing the access selection algorithm, few researchers have considered received signal strength (RSS) as one of the decision parameters for selecting the network. This decision parameter helps in choosing the access network that has the highest received signal strength. However, RSS based selection mechanism is easy to implementation and low complexity. This often causes the effect of serious Ping-Pong [8]. Some researchers focused on connecting the users to the network along with the least load to achieve the load balance. This type of algorithm does not consider the network link performance and the users are connected with low link quality thus, user requirements are not guaranteed [9].

Few researchers have considered the various decision parameters while designing the access selection algorithm such as energy consumption, service price, moving speed, packet loss ratio, jitter, delay, and network load, further it is modeled as the multiple attribute decision making (MADM) problem [10], [11]. Moreover, the MADM mechanism gathers data first for decision parameters and later it is normalized and all attributes are computed considering the decision parameters further candidate networks are ranked in order. It is observed that various users require various levels of satisfaction having the same decision parameter value with having various user diversity. Hence, few papers have used the utility theory for quantifying user satisfaction along with decision parameter [12]. Access selection mechanism based on the utility function, it is used for designing the various utility functions and absolute values are converted into the utility values for the given candidate network, rank in order and access network that has the highest value of utility. Also, few papers considered the game theory model [13], Markov Chain model [14], and optimized model [15] for developing the access selection algorithm.

The above discussed selection mechanisms are mainly based on the utility theory, RSS and MADM, these mechanisms require absolute decision parameter description and these decision parameters are difficult to be modeled in a heterogeneous wireless network (HWN) environment. Moreover, for adopting the HWN dynamic environment algorithms need to correct the operating parameters which further results in the intervention process of humans and restrict discussed algorithms [16]. Moreover, most of the existing protocols of RAT (mechanism [17]-[20] are developed for achieving quality of service (QoS) a network. However, very few mechanisms considered the RAT selection method by considering the HWN environment [21]-[24]. In the case of the radio access technology (RAT) selection mechanism it fails to consider multiple applications at the same time, these phenomena are known as multi-service multi-mode terminals (MMT). Furthermore, to address this issue, existing methodologies either consider user preference or service requirement. Hence, it fails to achieve the tradeoff between the user preference and application requirement. For addressing the research issues, this work presents a quality of experience aware RAT selection method for a highly dense and dynamic heterogeneous communication environment. The quality of experience RAT (QoE-RAT) selection method considers network attributes (QoS) and multi-mode terminal service preference requirements for selecting the best RAT among accessible RAT's under HWNs.

\section{QoE-RAT SELECTION MODEL FOR MULTIPLE CALLS AND MULTIMODE HETEROGENEOUS COMMUNICATION NETWORK}

This section presents a QoE-RAT selection model for multiple calls and multi-mode heterogeneous communication networks. First, the system model of enhanced recovery after thoracic surgery (ERAT) is presented. The proposed QoE-RAT selection method combines fuzzy computing [6] and MADM method for selecting suitable RAT for future communication. The algorithm flow of the proposed QoE-RAT is described in Figure1.

\subsection{System model for QoE-RAT}

This particular research considers the HWN which is described in Figure 2, in general HWN comprises long term evolution (LTE), WiMax, and universal mobile telecommunication (UMTS). The architecture of this model gives seamless mobility which is based on the environment of high mobile terminal density. Moreover, there are various scenarios for mobile users and further these users roam in different 
directions in the given signal coverage also these users are considered as the MMT that has the capability of processing the entire wireless technologies and can access any. Further, the network selection process is divided into three distinct stages which include network discovery, network selection decision making, access execution and also, it is presumed that users are capable of detecting and gathering the attribute data in a given range of networks. These users are also capable of ranking the candidate networks by given data as well as by selection protocol. This completes the network connection by the wireless network protocol. Moreover, this research work focuses on the five distinctive performance attributes i.e., bandwidth/data rate, latency, jitter, packet failure probability and service cost. Along with, as different multi-mode mobile terminal services have diverse sensitivity within the same RAT attribute (i.e., for data based services) require larger bandwidth, for video based services, the resolution of video is adjusted by bandwidth availability and in audio based service (i.e., calls) requires minimal bandwidth assurance and so on). Thus, this work, segments the multimode mobile terminal application services into the following category by application service features such as perfect elasticity (i.e., data based application service), partially elasticity (i.e., video based application service), and in elasticity (i.e., audio based application services). Along with, in this paper considers that end multi-mode mobile terminal uses any of these application services.

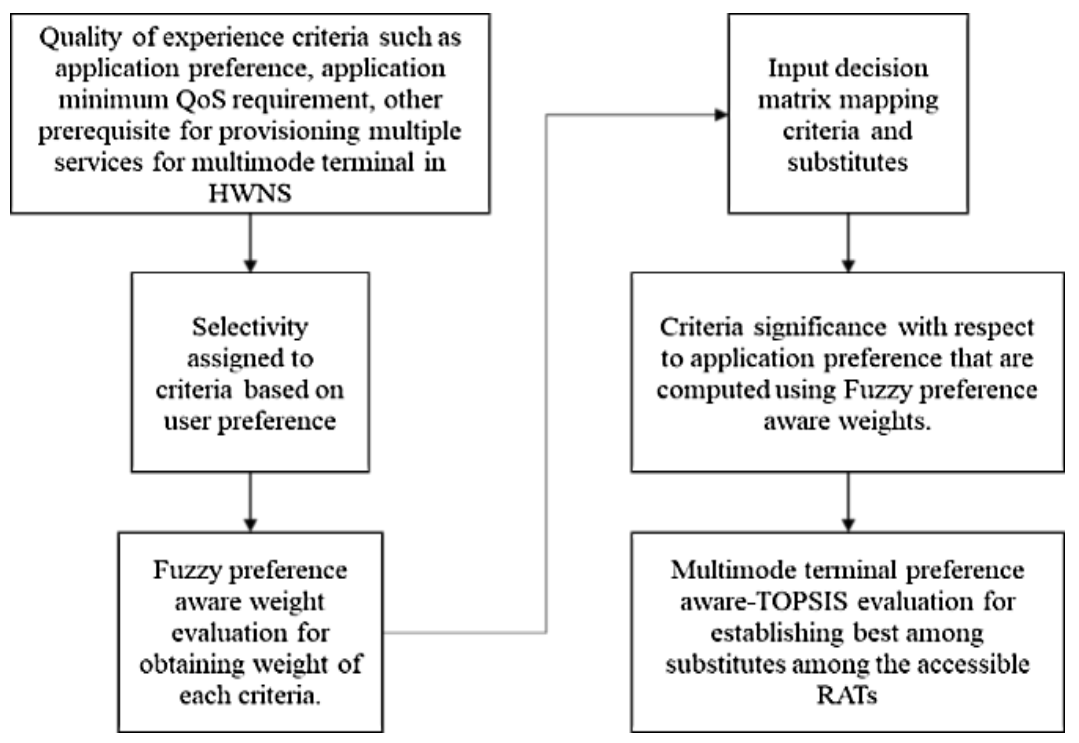

Figure 1. The flow diagram of proposed QoE-RAT selection method

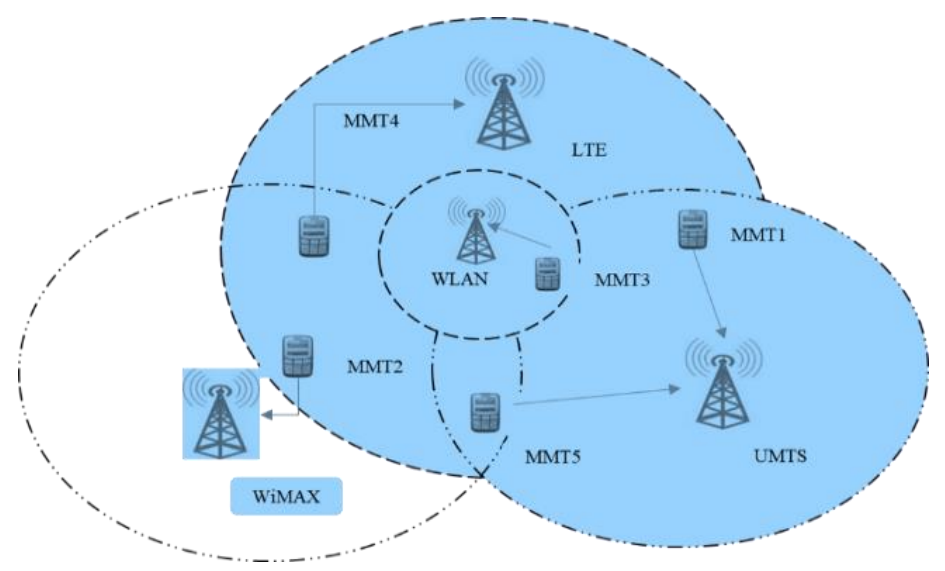

Figure 2. The architecture of heterogeneous wireless network

The proposed QoE-RAT selection method is composed of two phases. First, the criteria are weighted according to the communication network and user applications preferences. Second, decision-making (DM) is carried out in accordance with criteria and accessible to the RAT organization. In 
the first phase, the usefulness criteria are weighted in accordance with user application priority/selectivity is measured. The input pair-wise matrix (PM) is built in accordance with criteria with respect to criteria mapping using fuzzy preference aware weight [6], [25]. More detail of fuzzy preference-based weight evaluation is discussed in section $a$. Then, RAT ranking is carried out using the mapping of accessible RAT with respect to qualifying criteria that form the DM. Post that multi-mode terminal preference aware TOPSIS (MMTPA-TOPSIS) is modeled for obtaining ranking lists of RAT's. The rank list structure describes the ideal RAT when selecting it in descending order.

\subsection{Fuzzy preference aware weight evaluation method}

The mobile terminal contextual parameter of every service classes are represented as a weight that is given to respective network attributes. The mobile terminal context specific weight for different service can be described as (1),

$$
X_{h}^{t, V}=\left\{X_{h, k}^{t, V}\right\}
$$

where, $X_{h, k}^{t, V}$ depicts mobile terminal context specific weights of attribute $d_{k}$ considering respective service $t_{h}^{u}$ and $X_{h, k}^{t, V}$ are defined in range of [0,5], where 0 is given less importance and 5 is give higher importance. The mobile terminal defined selectivity parameter is represented as a vector for using (2),

$$
Q^{u, V}=\left\{q_{h}^{u, V}\right\}
$$

where, $q_{h}^{u, V}$ defines selectivity level of respective service $t_{h}^{u}$.

The prerequisite of QoE aware attributes weights and selectivity of application service are computed and used for choosing best RATS for respective multimode mobile terminals. However, the multimode mobile terminals can optimize these preference weight as per its requirement in dynamic manner. The model can establish the multimode mobile terminal quantified weights of HWNs attributes for respective collection of service using quantified selectivity of each service and respective attributes weights. The steps for computing MMT-quantified RAT attributes weights for respective collections of services is described below.

First normalization of mobile-terminal defined contextual weight vector $X_{h}^{u, V}$ of service $t_{h}^{u}$ using (4), (5). $X_{h}^{u, V}$ defines the qualified importance of network attribute defined by mobile terminal for respective service $t_{h}^{u}$ which can be mathematically represented as (3).

$$
X_{h}^{u, V}=\left\{x_{h, 1}^{u, V}, \ldots x_{h, k}^{u, V}, \ldots, x_{h, O}^{u, V}\right\}, h=1, \ldots, Z .
$$

The normalization operation of $X_{h}^{u, V}$ done using (4) and (5).

$$
\begin{aligned}
& \bar{X}_{h}^{u, V}=\left\{\bar{x}_{h, 1}^{u, V}, \ldots \bar{x}_{h, k}^{u, V}, \ldots, \bar{x}_{h, O}^{u, V}\right\} \\
& \bar{X}_{h}^{u, V}=\frac{x_{h, k}^{u, V}}{\sum_{k=1}^{O} x_{h, k}^{u, V}}
\end{aligned}
$$

Second, normalization of mobile terminal defined service selectivity vector $Q^{u, V}$ is done as (6).

$$
Q^{u, V}=\left\{q_{1}^{u, V}, \ldots, q_{h}^{u, V}, \ldots, q_{Z}^{u, V}\right\}, \quad h=1, \ldots, Z
$$

Normalize $Q^{u, V}$ for obtaining $\bar{Q}^{u, V}$ defined in (8).

$$
\begin{aligned}
\bar{Q}^{u, V} & =\left\{\bar{q}_{1}^{u, V}, \ldots, \bar{q}_{h}^{u, V}, \ldots, \bar{q}_{Z}^{u, V}\right\} \\
\bar{Q}^{u, V} & =\frac{q_{h}^{u, V}}{\sum_{h=1}^{Z} q_{h}^{u, V}}
\end{aligned}
$$

Third, this paper produces the normalization of weight vector $\bar{X}_{h}^{u, V}$ with respect to network attributes of different service and normalized selectivity vector $\bar{Q}^{u, V}$. Lastly, the mobile terminal defined weight vector $X^{V}$ of network attribute sets of respective service classes using (10). 


$$
\begin{aligned}
& X^{V}=\left\{x_{1}^{V}, \ldots, x_{k}^{V}, \ldots, x_{O}^{V}\right\} \\
& X^{V}=\sum_{h=1}^{Z} \bar{x}_{h, k}^{u, V} * \bar{q}_{h}^{u, V}
\end{aligned}
$$

Where, $\bar{q}_{h}^{u, V}$ defines the normalized selectivity outcomes of service $t_{h}^{u}, Z$ represent different kind service used by mobile terminal, and $\bar{x}_{h, k}^{u, V}$ defines normalized attribute weight $d_{k}$ for respective service $t_{h}^{u} \cdot x_{k}^{V}$ depicts attributes weight $d_{k}$ for service classes set defined by mobile terminal.

The proposed fuzzy preference aware weight calculation methods can handle fuzziness and uncertainty among fuzzy number or rule sets. Thus, this work uses fuzzy preference aware weight for computing the service-specific weights of HWNs attributes. The fuzzy preference aware weight builds different criteria (i.e., (fuzzy rules) for decision making problems. For representing the fuzziness of preferences this work uses triangular fuzzy numbers (TFNs). The TFN are represented as $N=(1, n, v), 1 \leq$ $n \leq v$, where $1, n, v$ represent the lower bounding parameter, the best promising parameter and the upper bounding parameter conveyed by the decision maker, respectively. The fuzzy rules will be a real number when $1=n=v$. The association among the significance of the TFN and the attributes are defined using (11) [26].

$$
\mu_{\tilde{O}(y)}= \begin{cases}\frac{y-1}{n-1} & m \leq y \leq n \\ \frac{v-1}{v-n} & n \leq y \leq v \\ 0 & \text { otherwise }\end{cases}
$$

The computation process of TFNs is described in (12), (13), and (14),

$$
\begin{aligned}
& N_{1}+N_{2}=\left(m_{1}+m_{2}, n_{1}+n_{2}, v_{1}+v_{2}\right) \\
& N_{1} \otimes N_{2}=\left(m_{1} * m_{2}, n_{1} * n_{2}, v_{1} * v_{2}\right) \\
& \frac{1}{N_{1}}=\left(\frac{1}{v_{1}}, \frac{1}{n_{1}}, \frac{1}{m_{1}}\right)
\end{aligned}
$$

where, $N_{1}$ and $N_{2}$ are two TFNs which is computed as (15).

$$
N_{1}=\left(m_{1}, n_{1}, v_{1}\right)
$$

and $N_{2}$ is computed as (16).

$$
N_{2}\left(m_{2}, n_{2}, v_{2}\right)
$$

The independent selectivity vector of multiple service $Q^{u, T}$ is defined using (17),

$$
Q^{u, T}=\left\{q_{h}^{u, T}\right\}, g=(1, \ldots, Z)
$$

where, $q_{h}^{u, T}$ defines the selectivity parameter of service $t_{h}^{u}$ among service classes established by service contextual features. For easiness in this work, it is assumed same as $Q^{u, V}$. Therefore, the selectivity vector after normalizing it can be established by service are described using (18).

$$
\bar{Q}^{u, T}=\left\{\bar{q}_{1}^{u, T}, \ldots, \bar{q}_{h}^{u, T}, \ldots, \bar{q}_{Z}^{u, T}\right\}
$$

The service-contextual attributes weight $X_{h}^{u, T},(g=1, \ldots, Z)$ of different service considered in HWNs can be computed using fuzzy preference aware weight, then fusion $X_{h}^{u, T}$ and $\bar{Q}^{u, T}$ for obtaining service-contextual attributes weights of different service classes. The step involved for establishing servicecontextual attribute weight calculation fuzzy preference are as follows. First, build a structure of parameter of multi-terminal and multi-service HWNs selection problems. Second, build fuzzy assessment matrix $B^{h}(h=1, \ldots Z)$ for services $t_{h}^{u}$ as stated in (19), $Z$ different type of service used in mobile terminal, and $o$ network attribute size considered $(o=O)$,

$$
B^{h}=\left(b_{j k}\right)_{o * o}
$$


where, $b_{j k}$ depicts significance of attribute $d_{j}$ with respect to $d_{k}$ considering service $t_{h}^{u}$. The $b_{j k}$ can be established as (20),

$$
b_{j k}=\left(m_{j k}, n_{j k}, v_{j k}\right)
$$

when $j \neq k, b_{j k}=1 / b_{j k}$, else $b_{j k}=(1,1,1)$. Third, compute the fuzzy interpretation $T_{j}$ of respective attribute $d_{j}$ with respect with $(21)$;

$$
S_{j}=\sum_{k=1}^{o} \alpha_{j k} \otimes \frac{1}{\left[\sum_{j=1}^{o} \sum_{k=1}^{o} \alpha_{j k}\right]}
$$

where,

$$
\left[\sum_{k=1}^{o} \alpha_{j k}\right]=\left(\sum_{k=1}^{o} m_{j k}, \sum_{k=1}^{o} n_{j k}, \sum_{k=1}^{o} v_{j k}\right)
$$

and

$$
\frac{1}{\left[\sum_{j=1}^{o} \sum_{k=1}^{o} \alpha_{j k}\right]}=\frac{1}{\sum_{j=1}^{o} \sum_{k=1}^{o} v_{j k}}, \frac{1}{\sum_{j=1}^{o} \sum_{k=1}^{o} n_{j k}}, \frac{1}{\sum_{j=1}^{o} \sum_{k=1}^{o} m_{j k}}
$$

and $T_{j}$ represent a TNF where $T_{j}$ is expressed as (24).

$$
T_{j}=\left(m_{j}, n_{j}, v_{j}\right)
$$

Then, compute likelihood $W\left(T_{k} \geq T_{j}\right)$ that fuzzy inference outcome $T_{k}$ is higher than $T_{j}$ as described in (25),

$$
W\left(T_{k} \geq T_{j}\right)=\left\{\begin{array}{cc}
1 & n_{k} \geq n_{j} \\
\frac{m_{k}-v_{j}}{\left(m_{k}-v_{j}\right)-\left(m_{k}-v_{j}\right)} n_{k} \leq & n_{j}, m_{j} \leq v_{k} \\
0 & \text { others }
\end{array}\right.
$$

where, $T_{k}$ and $T_{j}$ represent fuzzy inference outcomes of the attribute $d_{k}$ and $d_{j}$, respectively and $k \neq j$. Then, weight outcome $x_{h, k}^{u, T}$ of HWNs attribute $d_{k}$ for respective service $t_{h}^{u}$. For that this work compute the preliminary weight $x_{h, k}^{u, T \prime}$ of attributes $d_{k}$ as described in (26).

$$
x_{h, k}^{u, T \prime}=\min W\left(T_{k} \geq T_{j}\right)=\min W\left(T_{k} \geq T_{1}, T_{2}, \ldots, T_{O}\right), k=1, \ldots O
$$

After computing preliminary weights, it is normalized and $d_{k}$ is established by service $t_{h}^{u}$ satisfying $\sum_{k=1}^{o} x_{h, k}^{u, T}=1$ using (27).

$$
x_{h, k}^{u, T}=\frac{x_{h, k}^{u, T \prime}}{\sum_{k=1}^{o} x_{h, k}^{u, T \prime}} \quad k=1, \ldots, O
$$

The finalized HWN attribute weight vector $x_{h, k}^{u, T}=\left\{x_{h, 1}^{u, T}, x_{h, 2}^{u, T}, \ldots, x_{h, O}^{u, T}\right\}$ is established by service $t_{h}^{u}$ are obtained.

Lastly, fusion of HWNs attribute weight vector $s_{h}^{u}$ of different service and service selectivity vectors $\bar{Q}^{u, T}$ for obtaining HWNs attributes weight vector $X^{T}$ are established using service in mobile terminal as described in (28) and (29),

$$
\begin{aligned}
& X^{t}=\left(X_{1}^{T}, \ldots, X_{k}^{T}, \ldots, X_{O}^{T}\right) \\
& X_{k}^{T}=\sum_{h=1}^{Z} x_{h, k}^{u, T} * \bar{q}_{h}^{u, T}
\end{aligned}
$$


where, $x_{h, k}^{u, T}$ represent attribute weights $d_{k}$ established by service $t_{h}^{u}$ computed using (27), and $\bar{q}_{h}^{u, T}$ represent normalized selective outcomes of $t_{h}^{u}$, and $x_{k}^{t}$ depicts the attributes weight $d_{j}$ for different service class established by service contextual features. After establishing weight vector $X$ and the matrix $V$ for a different service class, the function of establishing suitable target RAT using multimode terminal preference aware technique for order of preference by similarity to ideal solution (TOPSIS) [27]. The proposed quality of experienced aware RAT selection method attains superior result when compared with existing RAT selection method which is experimentally shown below.

\section{RESULTS AND DISCUSSION}

This section presents a performance evaluation of the proposed QoE-RAT over the existing RAT selection method. The implementation and simulation parameter considered are described [22], [23], [25]. The IEEE 802.11 RAT is considered for UMTS, WiMAX, LTE, and WLAN. Three different kinds of services such as real-time polling service (RTPS), non-real-time polling service (NRTPS), and best effort service are considered for experiment analysis. Each access network is given equal priority, for evaluating the performance of QoE-RAT under a dynamic mobility environment. This work is used the IEEE 802.11 standard MAC designed in the society of infectious and tropical disease (SIMITS) simulator [28]. For modeling cellular network, the channel is composed of additive white Gaussian noise (AWGN). Then, multipath fading and log-normal shadowing models are used for modeling the path loss model. Lastly, power control is ideal. Then, IEEE 802.11 is used for modeling WLAN, Rayleigh channel model is used and bandwidth is set to 3-27 Mbps. The mobile subscriber is distributed uniformly random across the HWN environment. New mobile subscribers and HO subscribers will obey Poisson distribution. Finally, 3 types of services are considered which composed of $40 \%$ of NRTPS service, $30 \%$ of RTPS services, and $30 \%$ of BE services. The performance of QOE-RAT and the existing RAT selection method is evaluated in terms of throughput, the number of handovers, and packet loss rate.

\subsection{Throughput performance of QoE-RAT over existing RAT selection method}

This section presents the throughput performance attained by the proposed QoE-RAT selection method over the existing RAT selection method. Figure 3 shows the throughput outcome attained using the proposed QoE-RAT selection method over the existing RAT selection method [22], [23] considering various mobile terminals. The QoE-RAT improves throughput by $10.36 \%, 20.501 \%, 26.17 \%$, and $30.085 \%$ over the existing RAT selection method considering 50, 100, 150, and 200 mobile terminals respectively. As a result, it can be seen throughput increases with respect to an increase in mobile terminal size. This is because a greater number of packets are being transmitted in the network. An average throughput performance improvement of $21.78 \%$ is attained by the proposed QoE-RAT over the existing RAT selection method.

\subsection{Packet loss rate performance of QOE-RAT over existing RAT selection method}

This section present packet loss rate performance attained of proposed QoE-RAT selection method over existing RAT selection method. Figure 4 shows the packet loss rate outcome attained be proposed QoE-RAT selection method over existing RAT selection method [22], [23] considering varied mobile terminals. The QoE-RAT reduce packet loss in network by $81.87 \%, 70.87 \% 69.59 \%$, and $71.424 \%$ over existing RAT selection method considering 50, 100, 150, and 200 mobile terminals respectively. From result it can be seen packet loss rate increase with respect increase in mobile terminal size. This is because challenges exist in finding suitable substitute RAT for communication of dynamic mobile terminal. An average packet loss rate performance improvement of $73.44 \%$ is attained by proposed QoE-RAT over existing RAT selection method.

\subsection{Number of Handover performance of QOE-RAT over existing RAT selection method}

This section present number of handover performance attained of proposed QOE-RAT selection method over existing RAT selection method. Figure 5 shows the number of handover outcome attained be proposed QOE-RAT selection method over existing RAT selection methods [22], [23] considering varied mobile terminal. The QoE-RAT reduce number of handovers in network by $77.78 \%, 62.069 \%, 60.46 \%$, and $64.2 \%$ over existing RAT selection methods considering 50, 100, 150, and 200 mobile terminals respectively. From result it can be seen number of handovers increase with respect increase in mobile terminal size. This is because challenges exist in finding suitable substitute RAT for communication of dynamic mobile terminal. An average number of handover performance improvement of $66.12 \%$ is attained by proposed QoE-RAT over existing RAT selection method.

\subsection{Cost performance of QOE-RAT over existing RAT selection method}


This section present number of cost performance attained of proposed QOE-RAT selection method over existing RAT selection method. Figure 6 shows the service provisioning cost outcome attained be proposed QoE-RAT selection method over existing RAT selection methods [22], [23] considering varied mobile terminals. The QoE-RAT achieves much better profit by $61.766,68.89 \%, 72.13 \%$, and $92.5 \%$ over existing RAT selection methods considering 50, 100, 150, and 200 mobile terminals respectively. From result it can be seen cost incurred increase with respect to increase in mobile terminal size. This is because challenges exist in finding suitable substitute RAT for communication of dynamic mobile terminal. An average profit performance improvement of $73.82 \%$ is attained by proposed QOE-RAT over existing RAT selection method. From overall result attained it can be seen the proposed RAT selection is robust and scalable with respect to mobile terminal size in attaining good throughput, packet loss rate, cost, and handover performance.

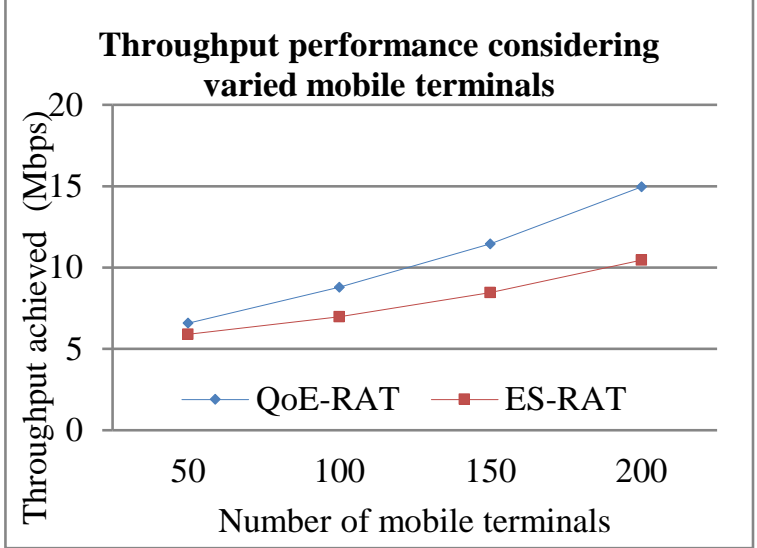

Figure 3. Throughput performance evaluation considering varied iteration

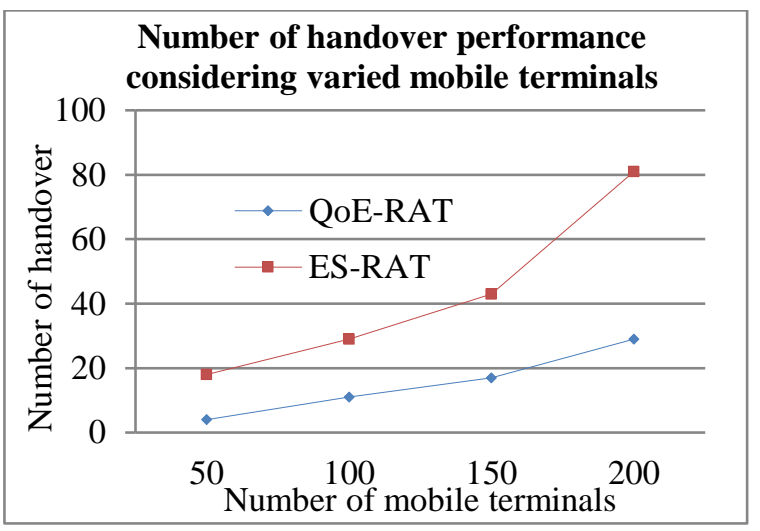

Figure 5. The Number of handover performance evaluation considering varied mobile terminal

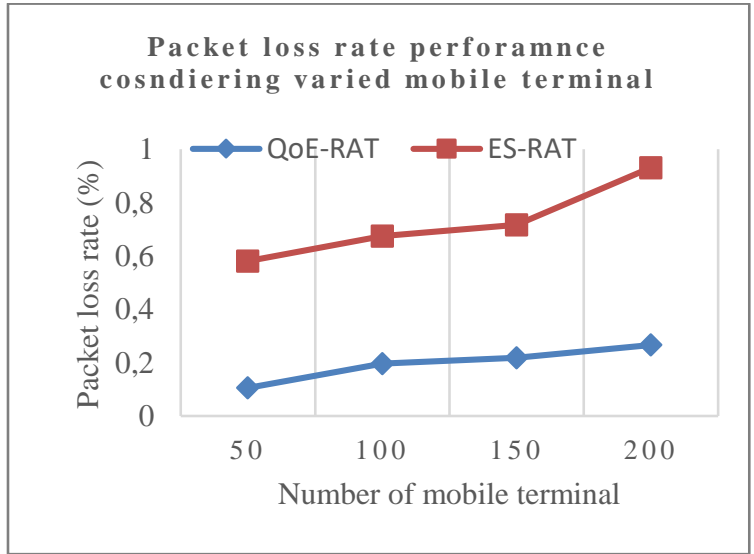

Figure 4. Packet loss rate performance evaluation considering varied mobile terminal

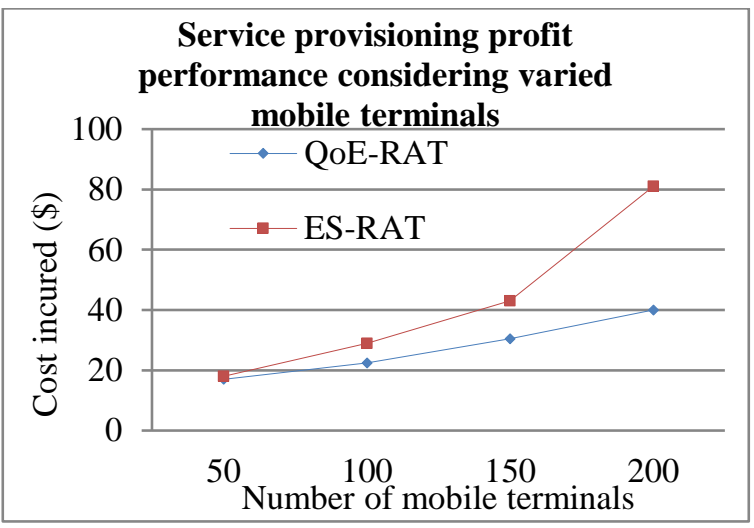

Figure 6. Cost performance evaluation considering varied mobile terminal

\section{CONCLUSION}

This paper first studied various existing RAT selection for HWN. From survey it is seen MADM with combination of Fuzzy, Markov decision (i.e., reinforcement leaning), deep learning are some methods used for RAT selection in multi-service multi-terminal HWNs. Most of existing RAT selection methods are designed without considering user QoE and preferences. Further, very limited work is carried out considering provisioning user preference considering multi-service HWN environment. This work presented fuzzy preference aware weight using multi-objective parameter for selection RAT in HWN. First, a decision matrix is built using fuzzy preference aware weight. Then, ideal RAT is selected using multimode terminal preference aware TOPSIS for carrying out handover operation and communication. Experiments are carried 
out to evaluate the performance of proposed QoE-RAT method over existing RAT selection method in terms of throughput, packet loss rate, cost, and number of handovers. From result attained it can be seen the QoE-RAT improves throughput performance by $21.78 \%$, improves packet loss rate performance by $73.44 \%$, improves handover performance by $66.12 \%$, and improves profit by $73.82 \%$ over existing RAT selection method considering varied mobile terminals under multi service HWN environment. From result attained it can be the QOE-RAT selection method attain superior QoE outcome when compared with existing RAT selection method. Future work would further considering developing efficient resource provisioning algorithm considering channel state information of user to utilize HWN resource more efficiently and evaluate performance under more dynamic mobility environment.

\section{REFERENCES}

[1] S. L. Poczter and L. M. Jankovic, "The Google Car: Driving toward a better future?," Journal of Business Case Studies (JBCS), vol. 10, no. 1, Art. no. 7, Dec. 2013, doi: 10.19030/jbcs.v10i1.8324.

[2] S. Islam, A. Hassan Abdalla Hashim, M. K. Hasan, and M. A. Razzaque, "MM-PNEMO: a mathematical model to assess handoff delay and packet loss," Bulletin of Electrical Engineering and Informatics (BEEI), vol. 8, no. 2, pp. 611-621, Jun. 2019, doi: 10.11591/eei.v8i2.1525.

[3] S.-C. Hung, H. Hsu, S.-M. Cheng, Q. Cui, and K.-C. Chen, "Delay guaranteed network association for mobile machines in heterogeneous cloud radio access network," IEEE Transactions on Mobile Computing, vol. 17, no. 12, pp. 2744-2760, Dec. 2018, doi: 10.1109/TMC.2018.2815702.

[4] A. El Hanjri, A. Hayar, and A. Haqiq, "Features detection based blind handover using kullback leibler distance for 5G HetNets systems," IAES International Journal of Artificial Intelligence (IJ-AI), vol. 9, no. 2, pp. 193-202, Jun. 2020, doi: 10.11591/ijai.v9.i2.pp193-202.

[5] A. Malik, J. Qadir, B. Ahmad, K.-L. Alvin Yau, and U. Ullah, "QoS in IEEE 802.11-based wireless networks: A contemporary review," Journal of Network and Computer Applications, vol. 55, pp. 24-46, Sep. 2015, doi: 10.1016/j.jnca.2015.04.016.

[6] M. Subramani and V. B. Kumaravelu, "A fuzzy based vertical handover network selection scheme for device-to-device communication," Indonesian Journal of Electrical Engineering and Computer Science (IJEECS), vol. 17, no. 1, pp. 324-330, Jan. 2020, doi: 10.11591/ijeecs.v17.i1.pp324-330.

[7] A. Keshavarz-Haddad, E. Aryafar, M. Wang, and M. Chiang, "HetNets selection by clients: Convergence, efficiency, and practicality," IEEE/ACM Transactions on Networking, vol. 25, no. 1, pp. 406-419, Feb. 2017, doi: 10.1109/TNET.2016.2587622.

[8] H. Kalbkhani, S. Yousefi, and M. G. Shayesteh, "Adaptive handover algorithm in heterogeneous femtocellular networks based on received signal strength and signal-to-interference-plus-noise ratio prediction," IET Communications, vol. 8, no. 17, pp. 3061-3071, Nov. 2014, doi: 10.1049/iet-com.2014.0230.

[9] Y. Li, B. Cao, and C. Wang, "Handover schemes in heterogeneous LTE networks: challenges and opportunities," IEEE Wireless Communications, vol. 23, no. 2, pp. 112-117, Apr. 2016, doi: 10.1109/MWC.2016.7462492.

[10] E. Obayiuwana and O. E. Falowo, "Network selection in heterogeneous wireless networks using multi-criteria decision-making algorithms: a review," Wireless Networks, vol. 23, no. 8, pp. 2617-2649, Nov. 2017, doi: 10.1007/s11276-016-1301-4.

[11] L. Tang, S. Ji, and J. Yan, "A heterogeneous network access selection algorithm based on attribute dependence," Wireless Personal Communications, vol. 92, no. 3, pp. 1163-1176, Feb. 2017, doi: 10.1007/s11277-016-3600-6.

[12] R. K. Goyal, S. Kaushal, and A. K. Sangaiah, "The utility based non-linear fuzzy AHP optimization model for network selection in heterogeneous wireless networks," Applied Soft Computing, vol. 67, pp. 800-811, Jun. 2018, doi: 10.1016/j.asoc.2017.05.026.

[13] S. Goudarzi, W. H. Hassan, M. H. Anisi, and S. A. Soleymani, "MDP-based network selection scheme by genetic algorithm and simulated annealing for vertical-handover in heterogeneous wireless networks," Wireless Personal Communications, vol. 92, no. 2, pp. 399-436, Jan. 2017, doi: 10.1007/s11277-016-3549-5.

[14] Y. K. Salih, O. H. See, and R. W. Ibrahim, "An intelligent selection method based on game theory in heterogeneous wireless networks," Transactions on Emerging Telecommunications Technologies, vol. 27, no. 12, pp. 1641-1652, Dec. 2016, doi: 10.1002/ett.3102.

[15] K. Ahuja, B. Singh, and R. Khanna, "Particle swarm optimization based network selection in heterogeneous wireless environment," Optik, vol. 125, no. 1, pp. 214-219, Jan. 2014, doi: 10.1016/j.ijleo.2013.06.011.

[16] S. Kubler, J. Robert, W. Derigent, A. Voisin, and Y. Le Traon, "A state-of the-art survey \&amp; testbed of fuzzy AHP (FAHP) applications," Expert Systems with Applications, vol. 65, pp. 398-422, Dec. 2016, doi: 10.1016/j.eswa.2016.08.064.

[17] M. Ozturk, M. Akram, S. Hussain, and M. A. Imran, "Novel QoS-aware proactive spectrum access techniques for cognitive radio using machine learning," IEEE Access, vol. 7, pp. 70811-70827, 2019, doi: 10.1109/ACCESS.2019.2918380.

[18] A. Zhu, S. Guo, B. Liu, M. Ma, J. Yao, and X. Su, "Adaptive multiservice heterogeneous network selection scheme in mobile edge computing," IEEE Internet of Things Journal, vol. 6, no. 4, pp. 6862-6875, Aug. 2019, doi: 10.1109/JIOT.2019.2912155.

[19] Q. Liu, T. Han, and N. Ansari, "Energy-efficient on-demand resource provisioning in cloud radio access networks," IEEE Transactions on Green Communications and Networking, vol. 3, no. 4, pp. 1142-1151, Dec. 2019, doi: 10.1109/TGCN.2019.2926287.

[20] A. Roy, V. Borkar, P. Chaporkar, and A. Karandikar, "Low complexity online radio access technology selection algorithm in LTE-WiFi HetNet," IEEE Transactions on Mobile Computing, vol. 19, no. 2, pp. 376-389, Feb. 2020, doi: 10.1109/TMC.2019.2892983.

[21] J. Ali et al., "Network selection in heterogeneous access networks simultaneously satisfying user profile and QoS," International Journal of Communication Systems, vol. 31, no. 13, Art. no. e3730, Sep. 2018, doi: 10.1002/dac.3730.

[22] J. Chen, Y. Wang, Y. Li, and E. Wang, "QoE-aware intelligent vertical handoff scheme over heterogeneous wireless access networks," IEEE Access, vol. 6, pp. 38285-38293, 2018, doi: 10.1109/ACCESS.2018.2853730.

[23] G. Liang and H. Yu, "Network selection algorithm for heterogeneous wireless networks based on service characteristics and user preferences," EURASIP Journal on Wireless Communications and Networking, vol. 2018, no. 1, Art. no. 241, Dec. 2018, doi: $10.1186 / \mathrm{s} 13638-018-1264-5$

[24] A. Ben Zineb, M. Ayadi, and S. Tabbane, "An enhanced vertical handover based on fuzzy inference MADM approach for heterogeneous networks," Arabian Journal for Science and Engineering, vol. 42, no. 8, pp. 3263-3274, Aug. 2017, doi: 10.1007/s13369-017-2418-1.

[25] S. M. Hussain, K. M. Yusof, R. Asuncion, and S. A. Hussain, "Artificial intelligence based handover decision and network 
selection in heterogeneous internet of vehicles," Indonesian Journal of Electrical Engineering and Computer Science (IJEECS), vol. 22, no. 2, pp. 1124-1134, May 2021, doi: 10.11591/ijeecs.v22.i2.pp1124-1134.

[26] M. Drissi, M. Oumsis, and D. Aboutajdine, "A multi-criteria decision framework for network selection over LTE and WLAN," Engineering Applications of Artificial Intelligence, vol. 66, pp. 113-127, Nov. 2017, doi: 10.1016/j.engappai.2017.08.019.

[27] N. G, H. S. Rameshbabu, and Gowrishankar, "User preference aware radio access technology selection model for heterogeneous communication network," Institute of Scholars (InSc).

[28] M. Manzano, F. Espinosa, Á. M. Bravo-Santos, and A. Gardel-Vicente, "Cognitive self-scheduled mechanism for access control in noisy vehicular Ad Hoc networks," Mathematical Problems in Engineering, vol. 2015, pp. 1-12, 2015, doi: $10.1155 / 2015 / 354292$

\section{BIOGRAPHIES OF AUTHORS}


Nagaraja Gadde (iD $8 \mathrm{SC}$ ( $\mathrm{P}$ is research scholar in the Department of Computer science and Engineering, Sai Vidya Institute of Technology, Bangalore, working as an Associate Professor department of Information Science and Engineering, SJC Institute of Technology, Chickballapur. Affiliated to Visveswaraya Technological University, Belagavi, India. He Completed BE in Computer Science and Engineering and M.Tech in Computer Science and Engineering. His area of interests includes wireless networks. He can be contacted at email: nagarajgadde11@gmail.com.

Basavaraj Jakkali (D) 8d SC P is Associate Professor in Department of Computer Science and Engineering, B.M.S. College of Engineering Bangalore. He Completed BE in Computer Science and Engineering and M.Tech in Computer Science and Engineering. His area of Interests includes wireless networks. His area of interests includes computer networks. He can be contacted at email: jakkali.cse@bmsce.ac.in.

Ramesh Babu Halasinanagenahalli Siddamallaih (iD IS SC P is Professor in Department of Computer science and Engineering, Sai Vidya Institute of Technology, Bangalore, Affiliated to Visveswaraya Technological University, Belagavi, India. He completed BE, MS and Ph.D. in Computer Science and Engineering. His area of interests includes wireless networks, wireless sensor networks, and internet of things. He has more than 23 years of academic experience. He can be contacted at email: rameshbabu.hs@saividya.ac.in.

Gowrishankar (D) SC P $\mathrm{P}$ is a senior Professor at computer Science and Engineering department at BMS College of Engineering, Bangalore. He served as a Head of the department of CS and E and IS \& E of BMSCE. He is actively associating in Research Collaborative Sabbatical program with University of Alabama, Huntsville (UAH), USA and he is a visiting professor for UAH. Having an Academic and Research experience of 20 years, he authored more than 80 research publications in reputed International journals and conferences with 150 citations in Scopus and Google scholar. His research interest includes performance evaluation, wireless network and deep learning. He guided $3 \mathrm{PhD}$ research scholars and currently guiding 8 research scholars leading to Ph.D. He is Chief coordinator of DATA CENTER, BMSCE since March 2009. Data Centre comprises of many state-ofthe-art software and hardware repositories. Under his leadership, department of computer Science and Engineering, has NBA accredited under teir-1 format. He can be contacted at email: gowrishankar@bmsce.ac.in. 